\title{
Participatory Design of a Virtual Reality Exercise for People with Mild Cognitive Impairment
}

Mahzar Eisapour

University of Waterloo

Waterloo, ON, Canada

eisapour@uwaterloo.ca

\section{Shi Cao}

University of Waterloo

Waterloo, ON, Canada

shi.cao@uwaterloo.ca

Laura Domenicucci

Schlegel Villages

Hamilton, ON, Canada

Laura.Domenicucci@schlegelvillages.com

\section{Jennifer Boger}

University of Waterloo /

Research Institute for Aging

Waterloo, ON Canada

jboger@uwaterloo.ca

Permission to make digital or hard copies of all or part of this work for personal or classroom use is granted without fee provided that copies are not made or distributed for profit or commercial advantage and that copies bear this notice and the full citation on the first page. Copyrights for components of this work owned by others than ACM must be honored. Abstracting with credit is

permitted. To copy otherwise, or republish, to post on servers or to redistribute to lists, requires prior specific permission and/or a fee. Request permissions

from Permissions@acm.org.

CHI'18 Extended Abstracts, April 21-26, 2018, Montreal, OC, Canada.

(C) 2018 Association for Computing Machinery.

ACM ISBN 978-1-4503-5621-3/18/04..\$15.00

https://doi.org/10.1145/3170427.3174362

\begin{abstract}
This case study describes the creation of a headmounted display virtual reality exergame program for promoting physical exercise for people with mild cognitive impairment $(\mathrm{MCl})$, namely people with earlystage dementia. We engaged in an iterative participatory design process with kinesiologists, recreational therapists, and people with $\mathrm{MCl}$ prior to pilot-testing a prototype program with three persons with $\mathrm{MCl}$. The test participants engaged in the exergame, were able to do the exercises, and their feedback was very positive. Engaging with professionals and people with dementia throughout the design process was very beneficial to creating a usable and engaging design as well as identifying areas that could be further improved. In conclusion, the approach illustrated through this case study resulted a new way for older adults with $\mathrm{MCl}$ to engage in physical activities that is fun and tailored to their abilities. The next phase in our research is to evaluate the exergame against comparable human-guided movements.
\end{abstract}

\section{Author Keywords}

Virtual Reality; Mild Cognitive Impairment; Dementia Exergame; Immersion; Physical Activity Motivation; Presence; User-centered Design. 


\section{ACM Classification Keywords}

H.5.m. Information interfaces and presentation (e.g., $\mathrm{HCl}$ ): Miscellaneous.

\section{I ntroduction}

Dementia refers to a category of neurocognitive disorders and symptoms that impair a person's cognitive abilities. Worldwide, the number of people living with dementia is estimated to be 35.6 million in 2010 and is expected to increase to 115.4 million by 2050 [7]. Living with dementia can make it more difficult to stay healthy and active, including safely engaging in physical exercise. The benefits of exercise for older adults is well established; studies have shown exercise results in improved fitness, improved physical and cognitive functioning, and an increase in positive behavior [6]. However, most exercise programs for older adults living in long-term care are verbally guided by physical therapists in a group setting. This method has several limitations. First, people with dementia often have impairments in language processing so may not be able to understand and follow the instructions. Second, they may lack the motivation to do it regularly [20]. Third, they may feel uncomfortable exercising in a group setting and therefore avoid group exercise classes.

Our goal is to create a virtual reality (VR) exercise program that is intuitive and engaging for older adults living with dementia. As the first step, we focused on people with mild cognitive impairment $(\mathrm{MCl})$, namely people with early-stage dementia. People with $\mathrm{MCl}$ are considered a good target population as they are in need of exercise support while having enough cognitive abilities intact to engage with the technology and researchers.
The current study highlights our use of user-centered design principles to create a VR exercise game that considers the needs and abilities of people with $\mathrm{MCl}$. We outline our design process, including engaging in participatory design with kinesiologists, recreational therapists, and people with $\mathrm{MCl}$.

\section{Background}

Researchers have studied the effects of different physical activities such as aerobic, cycling, and gait training with people with $\mathrm{MCl}$, and in general, the results showed beneficial effects in maintaining both physical and cognitive capabilities $[19,22]$. While exercise is beneficial, age-related changes often result in decreased physical activity for older adults, particularly those with $\mathrm{MCl}$ where changes in cognition create additional challenges $[5,15]$.

One method to promote physical exercise is to use interactive VR programs [12]. In general, VR refers to the simulation of a three-dimensional environment via any type of display device. Users interact with objects in VR using control devices such as joysticks and handheld controllers that can track hand position and orientation. VR programs for exercise purposes are often presented as games (i.e., exergames) that provide both guidance and enjoyment. For example, Nintendo Wii's sport games have been reported to promote exercise in older adults [3].

Head-mounted displays (HMDs) for VR are becoming widely available and affordable. HMD-VR uses headsets that have separate lens for each eye, creating an immersive three-dimensional experience. For example, a recent study presented a virtual biking activity for older adults using HMD-VR and demonstrated its 
feasibility and benefits including increased sense of presence [2]. In another study, HMD-VR has been used to improve fear of falling and balance issues for older adults [18]. We hypothesize that HMD-VR programs can also be designed for people with $\mathrm{MCl}$ to help promote physical exercise; however, research to support this hypothesis is limited. Previous studies of HMD-VR and $\mathrm{MCl}$ mainly focused on using VR as an assessment tool to evaluate people's cognitive capabilities and mental states in different activities such as cooking [8], mailing a letter [4], navigating in a simulated city center [17], and being interviewed by an avatar [9]. There is a lack of research focusing on using HMD-VR as a method to promote exercise for people with $\mathrm{MCl}$.

We designed and pilot-tested an immersive HMD-VR program for the promotion of physical activities for people with $\mathrm{MCl}$. This was done by leveraging our prior experience developing and assessing technologies for people with dementia $[1,10,11]$ as well as applying user-centered design and involving both people with $\mathrm{MCl}$ and professionals (kinesiologists and recreational therapists) throughout the design process. We have also created an experiment protocol that will use clinically meaningful indexes to compare the HMD-VR program with therapist-guided exercise; this protocol and the efficacy study will be reported in a future publication. In the remainder of the current report, we describe the participatory design process, the results from pilot-testing the program with three persons with $\mathrm{MCl}$, and the lessons learned.

\section{Observations and Focus Group}

The first step in the process was to gain an understanding of the abilities, preferences, and needs of the intended users (i.e., people with $\mathrm{MCl}$ ). We began by reviewing the user-centered design literature for guidelines regarding developing technology for people with $\mathrm{MCl}$. In addition to common usability principles for the general population $[13,14]$, we also consulted principles for older users [16,21]. A guiding premise is that the interface should be designed to be as simple and intuitive as possible to minimize users' workload on attention, comprehension, memory, and motor systems, as these are generally impaired for older adults. For example, in the 3D virtual world, visual objects should be high-contrast, large enough to be easily seen, and placed in front of the user in the central field of view. However, there is a lack of literature focusing on people with $\mathrm{MCl}$. Regardless, it is imperative for us as the designers to have first-hand knowledge of the characteristics and limitations of people living with $\mathrm{MCl}$.

We attended the recreational planning meeting held with 25 kinesiologists and recreational therapists from Schlegel Villages-a Canadian organization that manages 19 long-term care and retirement communities across Ontario. At the meeting, we presented the idea of HMD-VR and its use to engage older adults with $\mathrm{MCl}$. In general, they found the idea interesting; however, they also had a few concerns about the feasibility. For example, would people with $\mathrm{MCl}$ feel comfortable wearing the HMD? Would they feel disoriented during the transition between different VR scenes and between VR and the real world? Could they learn how to use the hand controllers to interact with virtual objects? Nevertheless, the caregivers were generally supportive. It was one of the attendees who suggested using HMD-VR to support exercise. It was also through this meeting that we met our exercise 


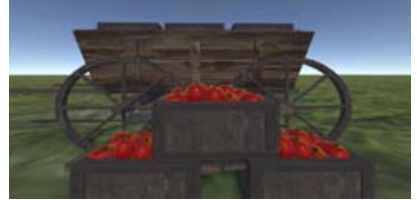

(a)

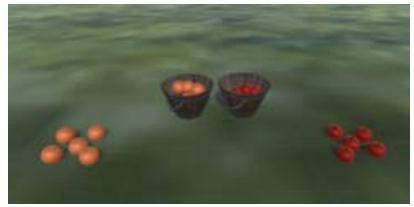

(b)

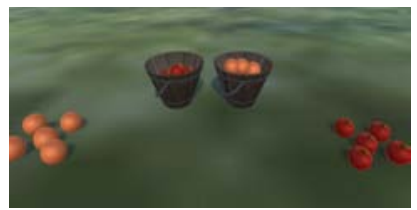

(c)

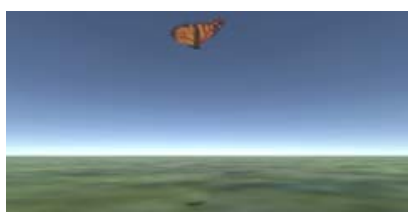

(d)

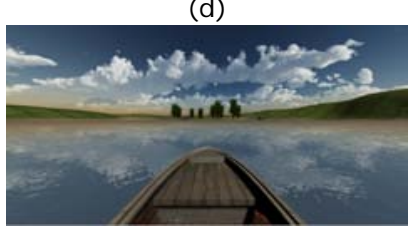

(e)

Figure 1: Different scenarios designed for the test a) apple box, b) straight ahead fruit sorting, c) cross-body fruit sorting, d) butterfly watching, e) rowing a boat. therapist team member, who has collaborated with us extensively throughout the project.

The three authors from University of Waterloo (two of whom had no previous experience designing with people with dementia) each conducted an eight-hour shadowing observation session in a long-term care center of Schlegel Villages. The goal was to gain firsthand experience about the life of people with dementia. During the sessions, the authors talked with the residents and participated in their activities such as walking, watching TV, and serving meals. It was observed that most residents had very limited physical activities. Although there were events planned for them such as singing, Tai Chi, puzzle solving, and towel folding, many residents sat and watched TV for much of the time. Many residents were also social and engaged when we interacted with them, which lent support to our expectation that the residents might enjoy VR programs if the programs can be designed properly for them to use.

The next stage involved a focus group meeting, where we brought a HMD-VR system (Oculus Rift CV1) and two existing VR programs for kinesiologists and people with $\mathrm{MCl}$ to try. The first program (Through the Ages: President Obama Celebrates America's National Parks) is a 360-degree VR movie that plays videos recorded from a national park. The second program (NVIDIA VR Funhouse) is a virtual carnival game with small games such as slicing balloons, whack-a-mole, and shooting plates, which require the use of hand controllers. Kinesiologists, therapists, and three residents with $\mathrm{MCl}$ tried using both VR programs; all of them could interact with the programs. This positive result helped improve our understanding of how people with $\mathrm{MCl}$ might react to HMD-VR and increased the research team's confidence. Valuable feedback was obtained from the discussion that followed. A kinesiologist commented that playing fighting games might agitate residents who had previous aggression issues. This suggested that calm activities should be used in our design. Another kinesiologist commented that hand gestures and buttons on the hand controllers were not very intuitive to learn and use. This device limitation suggested that our design should avoid interaction that requires specific gestures or pressing buttons. For safety reasons, we also decided that all users should play the VR programs while seated to avoid the risk of falling. This seating requirement is the same as the practice at Schlegel Villages for therapist-guided exercise.

\section{First I teration of Design and Testing}

In designing our VR exergame, we carefully and collaboratively selected appropriate motions, environment, and tasks. Because we have decided on the seated posture, the motions used in the exercise were limited to the upper body (i.e., neck, arms, and torso). Finger motions were excluded to increase simplicity and to allow users to keep holding the controllers in both hands. Applying these constraints, the exercise therapist on our team consulted with her therapist colleagues to select motions from the ones used in existing therapist-guided exercise. Through two design iterations (described below), five motions were selected to match the game scenario: 1) head rotation, 2 ) reaching straight ahead, 3) cross-body reaching, 4) lifting both arms, and 5) rowing with both hands.

Regarding the environment to implement the motions, we followed the therapists' recommendation to use a calm place that is enjoyable for most residents. The 


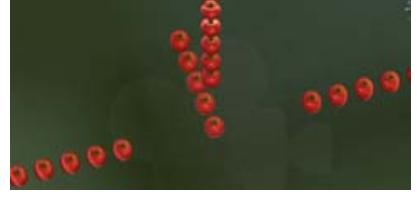

(a)

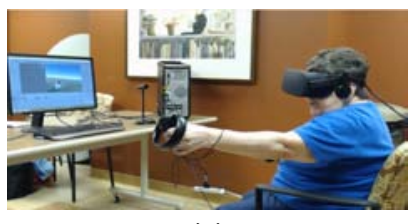

(b)

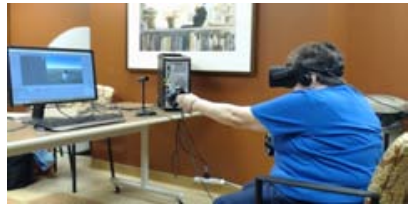

(c)

Figure 2: a) Calibration setup of objects in virtual environment using apples in different distance placed in the main

directions. b,c) a person following the calibration steps in two directions. place should be a common daily life location where the planned motions can be implemented as intuitive,

meaningful, and interesting activities. We considered a kitchen, a farm, a pet store, and a gym. Through many discussions with therapists, the farm was selected as it is familiar and interesting for most of the residents, is gender-neutral, and has a variety of activities that can be simulated.

We composed a list of candidate activities that were plausible to do in a farm and focus on upper body motions. The candidates included watching butterflies, picking flowers, grabbing fruits, turning wheels, painting fences, lifting boxes, and sorting various objects. Following the simplicity principle, we decided to only present a minimal number of objects in each activity to reduce cognitive workload. For example, most of the tasks should have only one interactive object in the scene. If selection was needed, no more than two options should be presented. In the end, the selected activities included butterfly watching (head and neck turning), sorting apples and oranges into baskets (arm reaching straight and across body), lifting a large object such as a box or a pile of hay onto a stand (lifting with both arms), and rowing a boat.

Next, we developed our first prototype HMD-VR exergame program. Based on our previous observations, literature review, and focus group discussion, the following specific design considerations were implemented to accommodate the needs of people with $\mathrm{MCl}$. The research team took care to clearly describe what was happening so the participants could feel more at ease, particularly with transitions. For example, "I am going to put this visor on your head and to show you a game. Would that be OK?" "Now, we're going to stop this game and take off the head set. Things might seem black for a bit." Researchers also conversed with the participants before the transition between different VR scenes. Task instructions were recorded as verbal guidance and played in the VR program. The instructions were read in a gentle, casual, and story-telling fashion. For example, “Wow, look! There are so many apples and oranges on the ground. How about you put the apples in the apple basket and the oranges in the orange basket?" For handling objects with the touch controller, the (virtual) target object was automatically placed in the (virtual) hand when the hand was touching the object, eliminating the need for button presses or gestures. After the object was moved to the target location, it was automatically removed from the hand and placed at the target location. In addition, all the visual objects were placed in the front field of view or close to it to minimize the need for searching and looking around.

We presented the initial prototype to a group of six kinesiologists/recreational therapists and four residents at Schlegel Villages for their comments and feedback. The residents responded very positively and enjoyed most of the activities very much. The kinesiologists commented that they were pleasantly surprised to see the level of engagement and degree of motion elicited by playing the game.

This preliminary testing was extremely valuable as it found several areas that could be improved and enabled in-person discussions with therapists and residents regarding how to go about improving them. For example, a pile of hay was initially used as the object in the object lifting task, but some residents had difficulty in finding an appropriate way to grab and lift it 
with both hands. After discussion, we decided to use a box instead and place two obvious handles on the ends to signal the places for holding it. Another issue that arose was the need for individual calibration. For example, in the apple sorting task, there was a considerable difference in residents' range of motion (ROM) capabilities. Customized object location is needed to avoid frustration, enable people to achieve their goals, and ensure that every user can benefit from an appropriate level of stretching.

\section{Second I teration of Design and Testing}

Based on feedback from therapists and people with $\mathrm{MCl}$, we revised the design and developed the second prototype, including the addition of a calibration initialization program. This is particularly important for older adults, who may have conditions that limit ROM (e.g., stroke, arthritis). Moreover, limitations in ROM may not be bi-lateral; a person may have significantly more ROM on one side than the other. During the calibration task, each user tried to reach targets (apples) presented at increasing intervals of distance along different directions (Figure 2). The ROM limit was recorded as a parameter for each individual person. This parameter was then used to automatically adjust object locations in the exergame with the activities calibrated to $80 \%$ of each individual's ROM limit to ensure that the exergame was well within their abilities.

The second prototype was tested with three persons with $\mathrm{MCl}$ using an Oculus Rift $\mathrm{CV} 1$, which has a resolution of 1080 by 1200 pixels per eye at 90 frames per second. Oculus touch controllers allowed the tracking of hand position and orientation. A digital camera was used to record participants' interaction with the program. During the test, participants were seated in a chair to avoid falling, and sufficient space was provided to avoid any collisions while using the HMD (Figure 3). An LCD monitor was used to mirror the participants' view inside the HMD for researchers in the same room to see and control the task procedures and detect any problems. The location of the test was at the Village of Wentworth Heights, which is a long-term care/retirement home in Hamilton, Ontario, Canada. All tests were supervised by two trained therapists to ensure the safety and wellbeing of participants. All three participants with $\mathrm{MCl}$ were naive to VR and the tasks. Participants each did the calibration task before trying the five exergame scenarios.

\section{Findings}

The calibration worked well, making objects reachable and close to the ROM limit for each person. In general, the response was very positive. All three participants could complete all the exergame tasks. In fact, therapists commented that the game was eliciting greater ROM than they thought some of the participants were capable of. While some were hesitant to try the HMD-VR, participants clearly enjoyed using it, including the calibration task.

All scenarios were engaging and enjoyable for the participants. For example, one of the participants seeing the apples in the calibration procedure said, "Oh wow, it's good! Can I take a bite of the apple?" Another participant, when she found herself in the boat rowing scenario, said "Oh, I am in a rowboat!" and started singing. For all three participants, the test was ended by the researchers (as we set a 15-minute time limit) rather than by the participants. 
Feedback was obtained from both people with $\mathrm{MCl}$ and therapists. For example, it was recommended to add more background objects such as trees and barns, which could help participants better understand that they are on a farm. In the butterfly watching task, it was recommended to add the calibration of both the range and speed of the butterfly's movement, accommodating people with different head and neck ROM. In the box lifting task, the height of the wagon should be adjusted so that participants can see the top of it, which can serve as a natural cue for the target location. The handles on the boxes should be larger and use a more salient color. In the boat rowing scenario, a recommendation was to add water sound. It was also recommended to add vibration on hand controllers as feedback to inform the completion of an action. We will consider and implement these valuable recommendations in our follow-up studies.

\section{Discussion}

Through our participatory design process creating an exergame, we have identified the following HMD-VR design recommendations for users with $\mathrm{MCl}$.

- Keep visual targets within the front field of view. Avoid searching that requires head motion.

- Provide verbal prompts before the transition between real world and VR, as well as the transition between different VR scenes.

- Provide verbal instructions that bring users into the scene and task, in a gentle, casual, and storytelling fashion.

- Avoid using buttons or gestures as control input. When moving objects, directly attach objects to the hand when it is reached, and directly remove objects from the hand at target locations.

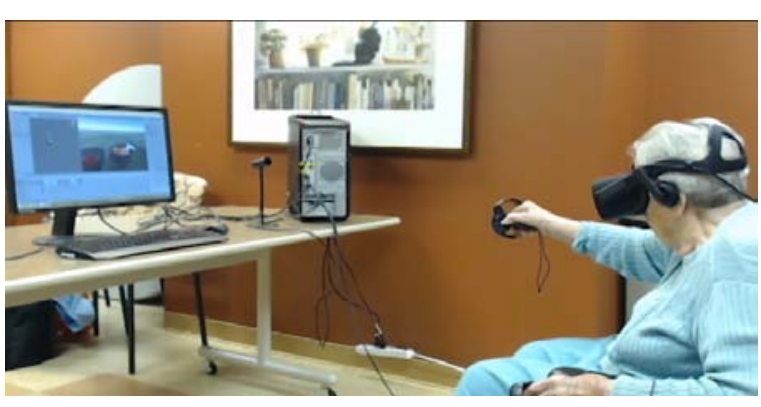

(a)

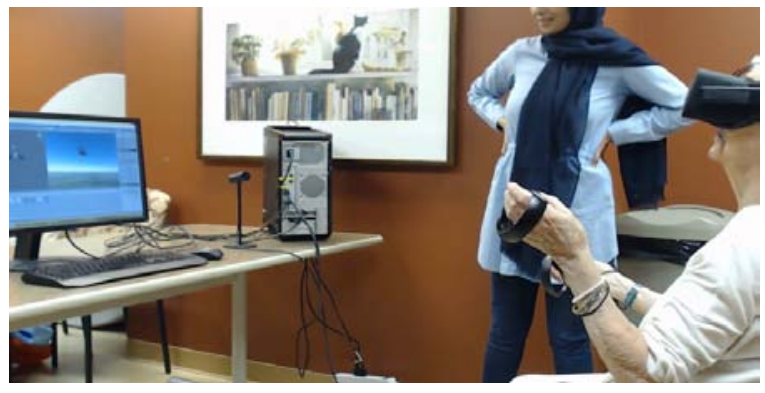

(b)

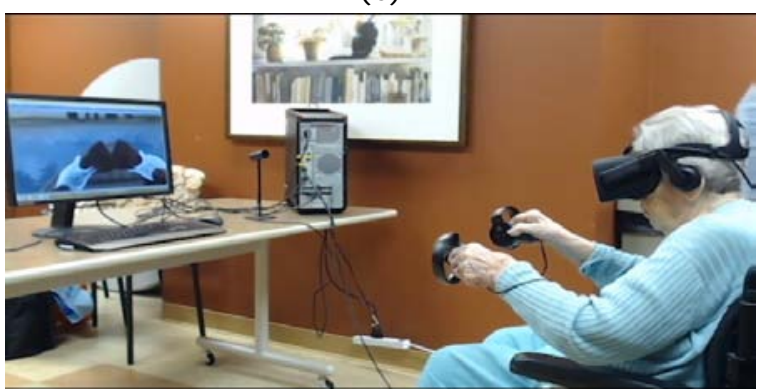

(c)

Figure 3: A person with $\mathrm{MCl}$ a) sorting apples and oranges into baskets, b) watching a butterfly, and c rowing a boat in the HMD-VR program. 
- Use high contrast and tasks that implicitly signify how to interact with objects (e.g., salient handles that indicate where to grab and hold).

- Use a calibration process to adjust the required range of motion for each individual; this ensures an accessible and engaging experience.

- Prevent errors, enable exploration of the game space in a supportive fashion, and clearly indicate when a task has been successfully accomplished.

- Consult specialists and get feedback from people with $\mathrm{MCl}$; participatory design process helps identify hidden problems and improve the quality of the VR program for the target users.

Interacting with virtual objects without using any buttons on the control interface is highly recommended for people with $\mathrm{MCl}$. In addition, because of individual difference in ROM and functional limitation, it is important to use a flexible design with calibration to customize the target distance for each individual.

\section{Conclusion \& What's next}

Through the participatory design process and pilot testing with caregivers and people with $\mathrm{MCl}$, we have developed a HMD-VR exergame for promoting physical exercise for people with $\mathrm{MCl}$. The extensive involvement of people with $\mathrm{MCl}$ and experts in exercise therapy for older adults was crucial to creating an exergame that was enjoyable and accessible by people with $\mathrm{MCl}$. While controlled experiment is needed to evaluate our exergame, the current results are promising, potentially offering a new way for older adults with $\mathrm{MCl}$ to engage in physical activity. As we do so, we must be considerate of people with $\mathrm{MCl}$ and carefully consider when and how such interventions are designed and used. The next phase in our research is to evaluate the exergame against comparable humanguided movements.

\section{Acknowledgement}

We gratefully acknowledge the time and input of our collaborators - experts in kinesiology and recreational therapy and people living with dementia.

\section{References}

1. Jennifer Boger, Babak Taati, and Alex Mihailidis. 2016. Interdisciplinary development of manual and automated product usability assessments for older adults with dementia: lessons learned. Disability and Rehabilitation: Assistive Technology 11, 7: 581-587.

2. Jon Ram Bruun-pedersen, Stefania Serafin, and Lise Busk Kofoed. 2016. Going Outside While Staying Inside - Exercise Motivation with Immersive vs. Non - Immersive Recreational Virtual Environment Augmentation for Older Adult Nursing Home Residents. IEEE International Conference on Healthcare Informatics (ICHI), 216226.

3. Ying-Yu Chao, Yvonne K Scherer, and Carolyn A Montgomery. 2015. Effects of using Nintendo Wii ${ }^{\mathrm{TM}}$ exergames in older adults: a review of the literature. Journal of aging and health 27, 3: 379402.

4. Darren Flynn, Paul van Schaik, Tim Blackman Clive Femcott, Brian Hobbs, and Carlos Calderon 2003. Developing a virtual reality-based methodology for people with dementia: a feasibility study. Cyberpsychology \& behavior: the impact of the Internet, multimedia and virtual reality on behavior and society 6, 6: 591-611.

5. Rebeca I Garcia-Betances, Viveca Jiménez-Mixco, Maria T Arredondo, and Maria F Cabrera-

Umpiérrez. 2015. Using virtual reality for cognitive training of the elderly. American J. of Alzheimer's Disease \& Other Dementias30,1:49-54. 
6. Patricia Heyn, Beatriz C Abreu, and Kenneth J Ottenbacher. 2004. The effects of exercise training on elderly persons with cognitive impairment and dementia: a meta-analysis. Archives of physical medicine and rehabilitation 85, 10: 1694-1704.

7. Julie Hugo and Mary Ganguli. 2014. Dementia and cognitive impairment: epidemiology, diagnosis, and treatment. Clinics in geriatric medicine 30, 3: 421-442.

8. Valeria Manera, Pierre-David Petit, Alexandre Derreumaux, et al. 2015. "Kitchen and cooking", a serious game for mild cognitive impairment and Alzheimer's disease: a pilot study. Frontiers in Aging Neuroscience 7, March: 24

9. Mario F Mendez, Aditi Joshi, and Elvira Jimenez. 2015. Virtual reality for the assessment of frontotemporal dementia, a feasibility study. Disabil Rehabil Assist Technol 10, 2: 160-164.

10. Alex Mihailidis, Scott Blunsden, Jennifer Boger, et al. 2010. Towards the development of a technology for art therapy and dementia: Definition of needs and design constraints. The Arts in Psychotherapy 37, 4: 293-300.

11. Alex Mihailidis, Jennifer N Boger, Tammy Craig, and Jesse Hoey. 2008. The $\mathrm{COACH}$ prompting system to assist older adults with dementia through handwashing: An efficacy study. BMC geriatrics 8, 1: 28.

12. Kimberly J Miller, Brooke S Adair, Alan J Pearce, Catherine M Said, Elizabeth Ozanne, and Meg M Morris. 2013. Effectiveness and feasibility of virtua reality and gaming system use at home by older adults for enabling physical activity to improve health-related domains: a systematic review. Age and ageing 43, 2: 188-195.

13. Jakob Nielsen. 1994. Enhancing the explanatory power of usability heuristics. Proceedings of the SIGCHI conference on Human Factors in Computing Systems, 152-158.

14. Don Norman. 2013. The design of everyday things: Revised and expanded edition. Basic Books (AZ).
15. Hanna Öhman, Niina Savikko, Timo E Strandberg, and Kaisu H Pitkälä. 2014. Effect of physical exercise on cognitive performance in older adults with mild cognitive impairment or dementia: a systematic review. Dementia and geriatric cognitive disorders 38, 5-6: 347-365.

16. Kara Pernice and Jakob Nielsen. 2008. Web usability for senior citizens: design guidelines based on usability studies with people age 65 and older. Nielsen Norman Group.

17. Paul Van Schaik, Anthony Martyr, Tim Blackman, and John Robinson. 2008. Involving persons with dementia in the evaluation of outdoor environments. CyberPsychology \& Behavior 11, 4: 415-424.

18. Daniel Schoene, Stephen R Lord, Kim Delbaere, Connie Severino, Thomas A Davies, and Stuart T Smith. 2013. A Randomized Controlled Pilot Study of Home-Based Step Training in Older People Using Videogame Technology. PLOS one 8, 3: 1-8.

19. Sandra Trautwein, Andrea Scharpf, Bettina Barisch-Fritz, Christina Niermann, and Alexander Woll. 2017. Effectiveness of a 16-Week Multimoda Exercise Program on Individuals With Dementia: Study Protocol for a Multicenter Randomized Controlled Trial. J MIR research protocols 6, 3 .

20. Ann K Williams. 2005. Motivation and dementia. Topics in Geriatric Rehabilitation 21, 2: 123-126.

21. Natalie E Wolfson, Thomas M Cavanagh, and Kurt Kraiger. 2014. Older adults and technology-based instruction: Optimizing learning outcomes and transfer. Academy of Management Learning \& Education 13, 1: 26-44.

22. Guohua Zheng, Rui Xia, Wenji Zhou, Jing Tao, and Lidian Chen. 2016. Aerobic exercise ameliorates cognitive function in older adults with mild cognitive impairment: a systematic review and meta-analysis of randomised controlled trials. $\mathrm{Br} \mathrm{J}$ Sports Med 50, 23: 1443-1450. 